\title{
The microbiome in rheumatology: Where are we and where should we go?
}

\author{
Julia Manasson 지, Rebecca B Blank, Jose U Scher
}

\section{Handling editor Josef S} Smolen

Division of Rheumatology, Department of Medicine, New York University Grossman School of Medicine, New York, New York, USA

\section{Correspondence to} Dr Jose U Scher, Division of Rheumatology, Department of Medicine, New York University Grossman School of Medicine New York, NY 10016, USA; jose.scher@nyulangone.org

Received 24 February 2020

Revised 14 April 2020

Accepted 15 April 2020

Published Online First

24 April 2020
Check for updates

(c) Author(s) (or their employer(s)) 2020. No commercial re-use. See rights and permissions. Published by BMJ.

To cite: Manasson J

Blank RB, Scher JU.

Ann Rheum Dis

2020:79:727-733

\section{ABSTRACT}

From birth, humans coexist and coevolve with trillions of micro-organisms inhabiting most body surfaces and cavities, referred to as the human microbiome. Advances in sequencing technologies and computational methods have propelled the exploration of the microbiome's contribution to human health and disease, spearheaded by massive efforts such as the Human Microbiome Project and the Europe-based MetaHit Consortium. Yet, despite the accumulated body of literature and a growing awareness among patients, microbiome research in rheumatology has not had a key impact on clinical practice. Herein, we describe some of the landmark microbiome studies in autoimmunity and rheumatology, the challenges and opportunities of microbiome research and how to navigate them, advances in related fields that have overcome these pitfalls, and future directions of harnessing the microbiome for diagnostic and therapeutic purposes.

\section{INTRODUCTION}

Humans have coevolved with and harbour trillions of microbes from the time they are born. This collection of symbiotic, commensal and pathogenic micro-organisms (and their genomes) found on the skin, mucosal surfaces and other organs is referred to as the human microbiome, a term coined by the Nobel laureate, Joshua Lederberg (figure 1). ${ }^{1}$ Although mostly resilient during the adult life, the composition of the microbiome can be influenced by numerous factors, including genes, age, sex, mode of delivery, nursing, diet, drugs (prescription and non-prescription), and pets, to name a few. ${ }^{23}$ Previously, the ability to define these microbial communities was limited to classic culturing techniques, which were able to isolate up to $20 \%$ of the microbes known today. ${ }^{4}$ This situation dramatically changed with the democratisation of nextgeneration sequencing technologies, which allowed for a higher proportion of organisms to be identified, including bacteria, fungi and viruses.

Through the use of these technologies and advanced computational analytics, we are now able to obtain an enormous amount of sequence data in each run, and have an efficient way of profiling microbial communities from different sources. The typical workflow involves obtaining samples of interest, isolating DNA from these samples and performing DNA sequencing of microbial taxa. This is done by either querying a marker gene, such as $16 \mathrm{~S}$ rRNA for bacteria or internal transcribed spacer (ITS) for fungi, or looking at the DNA in its entirety via shotgun metagenomic sequencing, which allows for the characterisation of gene families and pathways. ${ }^{3}$ With the development of more sophisticated modalities, we now have the ability to profile gene expression (metatranscriptomics), proteins (metaproteomics) and metabolites (metabolomics) collectively produced by the microbiota. ${ }^{56}$

In 2007 the National Institutes of Health (NIH) launched the landmark Human Microbiome Project (HMP), ${ }^{7}$ which was followed by the Europe-based MetaHit Consortium, ${ }^{8}$ two massive multicentre efforts that have propelled research on how microbes contribute to human health and disease (figure 1). In fact, this work revealed that the microbiome also plays a prominent role in autoimmune disease, as evidenced by the exponential growth of studies in virtually all realms of rheumatology over the last few years. ${ }^{9}$ Still, despite the extent and wealth of information that such a body of literature has yielded, microbiome research is yet to have a significant influence on clinical practice (eg, enabling drug discovery or patient stratification). ${ }^{10}$ In fact, some of the results have been correlative in nature, hampering their translation into the clinical setting. However, due to its popularity, discussion of the microbiome has percolated into conversations with patients, and in some cases has resulted in dietary and behavioural modifications. Here, we describe the advances in rheumatology, enumerate the type of research that should be pursued and what should generally be avoided, review how other disciplines have overcome these challenges, and look into the microbiome-based diagnostic and therapeutic pipeline.

\section{What is known about the microbiome in autoimmune diseases}

Numerous studies of animal models and humans have demonstrated a link between the microbiome and autoimmunity. For instance, a wide range of animal models for inflammatory arthritis remain healthy when raised under germ-free conditions, only developing their respective phenotypes when exposed to particular microbes. Examples include the HLA-B27 transgenic rat, ${ }^{11}$ the interleukin (IL) 1 receptor antagonist knockout (IL1rn-/-) mouse, ${ }^{12}$ the $\mathrm{K} / \mathrm{BxN} \mathrm{T}$ cell receptor transgenic mouse (which expresses the KRN T cell receptor transgene and the MHC class II molecule $\left.\mathrm{A}^{\mathrm{g} 7}\right)^{13}$ and the SKG mouse (which has a mutation in the Src homology 2 domain of zeta-chain-associated protein kinase 70 [ZAP-70]). ${ }^{14}$ These data have confirmed and validated the fact that intestinal microbiota are indeed required to trigger and drive systemic autoimmunity.

Similarly, studies of human autoimmune disease consistently characterise a state of microbial dysbiosis, a disruption in the homoeostasis of microbial communities and composition. ${ }^{15}$ Perhaps the most established connection is in rheumatoid arthritis (RA), where microbes were identified as potential 

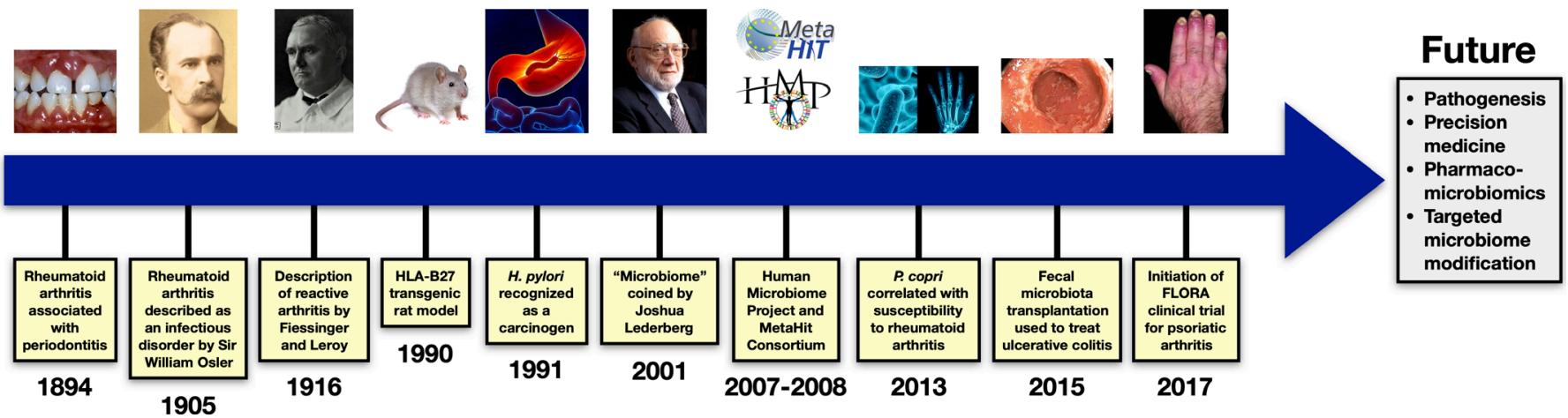

Figure 1 Historical time line of key events in microbiome research in rheumatology. Photo acknowledgements: 1894: https://www.efp.org/patients/ what-is-periodontitis.html; 1905: https://www.acponline.org/about-acp/about-internal-medicine/sir-william-osler-and-internal-medicine; 1916: https:// fr.wikipedia.org/wiki/No\%C3\%ABI_Fiessinger; 1990: https://www.dreamstime.com/stock-photos-grey-rat-isolated-white-image30500173; 1991: https://www.medicinenet.com/helicobacter_pylori/article.htm; 2001: https://www.nationalmedals.org/laureates/joshua-lederberg; 2007-2008: http:// gutcyc.org/aboutGutCyc; 2013: https://www.theatlantic.com/health/archive/2015/01/joint-pain-from-the-gut/383772/; 2015: https://www.gponline. com/clinical-review-ulcerative-colitis-epidemiology-diagnosis-management/gi-inflammatory-bowel-disease/ulcerative-colitis/article/1293409; 2017: https://www.merckmanuals.com/home/bone,-joint,-and-muscle-disorders/joint-disorders/psoriatic-arthritis.

contributors to disease as early as the 19th century. Sir William Osler attributed RA to tuberculosis, ${ }^{16}$ the toxaemic factor theory proposed that gut micro-organisms produced substances responsible for joint inflammation, ${ }^{17}$ and a connection between RA and periodontitis (PD) was established as early as 1894 (figure 1). ${ }^{18}$ More recently, Porphyromonas gingivalis, an oral anaerobe involved in the development of PD, was found to be highly prevalent in patients with RA, ${ }^{19}$ demonstrating positive serological correlation with anticitrullinated peptide antibody titres, ${ }^{20} 21$ considered to be triggers for RA. Interestingly, peptidylarginine deaminase 4 , an enzyme that catalyses citrullination, has also been identified as a contributor to RA pathogenesis. ${ }^{22-25}$ Distinct microbial perturbations exist in the gut as well, with expansion of Prevotella copri in untreated patients with new-onset RA, ${ }^{26} \mathrm{a}$ microbe that can induce an intestinal Th17 response leading to severe arthritis in SKG mice (figure 1). ${ }^{27}$ Evidence for P. copri involvement in pathogenesis derives from the identification of reactivity against a $P$. copri peptide in human RA and an associated Th1 response, ${ }^{28}$ providing a plausible mechanistic link.

The gut-joint axis is likewise involved in several conditions under the spondyloarthritis $(\mathrm{SpA})$ umbrella classification. In the HLA-B27 transgenic rat model of SpA there is a marked intestinal dysbiosis, ${ }^{29} 30$ which is linked to enhanced expression of Th1 and Th17 cytokines, expansion of Th17 cells in the colonic mucosa, and increased production of bacteria-specific $\operatorname{IgA}{ }^{30}$ These perturbations are highly dependent on host genetic background. ${ }^{31}$ In humans, the most obvious examples of the gut-joint connection are reactive arthritis, described in 1916 by Noël Fiessinger and Emile Leroy, ${ }^{32}$ which results after infection by enteropathogenic (and uropathogenic) bacteria, ${ }^{33}$ and enteropathic arthritis, described in 1929 by J Arnold Bargen, ${ }^{34}$ which is a sequala of inflammatory bowel disease (IBD) and can parallel colitis exacerbations (figure 1). ${ }^{35}$ Another example is ankylosing spondylitis, where terminal ileal biopsies demonstrate distinct microbial signatures driven by several core families of bacteria. ${ }^{36}$ In paediatric enthesitis-related arthritis, a type of juvenile SpA, there is reduction of intestinal Faecalibacterium prausnitzii (similar to IBD), ${ }^{37}$ a gut commensal that produces the shortchain fatty acid (SCFA) butyrate, ${ }^{38}$ which is known to promote the expansion of T-regulatory cells. ${ }^{39}$ In psoriatic arthritis (PsA), our group has demonstrated a significant reduction of two gut commensals, Akkermansia and Ruminococcus, correlating with higher levels of intestinal secretory IgA and lower levels of receptor activator of nuclear factor $\kappa-\mathrm{B}$ ligand, as well as reduction of local anti-inflammatory medium-chain fatty acids (MCFAs), hexanoate and heptanoate. ${ }^{40}$

Systemic lupus erythematosus (SLE) is also characterised by microbial perturbations. One of the earliest investigations showed decreased gut bacterial diversity and a lower Firmicutes:Bacteroidetes ratio in patients with SLE. ${ }^{41}$ Other relatively small studies have also demonstrated gut bacterial dysbiosis in SLE ${ }^{42-48}$ mostly corroborating previous findings. Intriguingly, similar to RA, translocation of intestinal pathobionts was likewise noted with the description of Enterococcus gallinarum in liver biopsies of patients with SLE. ${ }^{49}$ Furthermore, new evidence in mice and humans suggests that translocation of commensal orthologs of a human autoantigen, such as Ro60, can drive autoimmunity and may be a factor in SLE pathogenesis. ${ }^{50}$

There is far less evidence for microbiome involvement in systemic sclerosis. The majority of research to date has characterised gut microbial communities in those with and without disease, ${ }^{51-55}$ focusing on the dysbiosis in patients with gastrointestinal features of systemic sclerosis. Many of the studies found decreased gut bacterial diversity and lower levels of 'antiinflammatory' microbiota such as Bacteroides, Faecalibacterium and Clostridium, as well as higher levels of pathobionts such as Fusobacterium. ${ }^{52-55}$

Navigating and addressing challenges in microbiome research As discussed, although murine work has established a definite role for microbiome contribution to rheumatological conditions, the majority of human studies have been correlative in nature and therefore unable to distinguish whether dysbiosis precedes disease onset or is rather a consequence of the inflammatory process. Furthermore, very few studies propose a mechanism for pathogenesis. In general, microbiome experiments require thoughtful and thorough planning in order to generate data that are robust and reproducible, enabling their applicability in the clinic (table 1). Starting with experimental design, in lieu of performing exploratory correlative studies, the field should aim to answer particular questions that will preferably have a direct impact on patient care. In planning a microbiome experiment, it is also critical to select the appropriate control population and account for factors that may influence or confound the data, including host genes, ${ }^{5657}$ age $^{5859}$ and sex, ${ }^{60}$ as well 
Table 1 Dos and don'ts in microbiome research

\begin{tabular}{ll}
\hline Dos & Don'ts \\
\hline Specific hypothesis designed to answer a clinically relevant question & Exploratory correlative surveys \\
\hline Appropriate control populations & No control population \\
\hline Incorporation of factors that influence microbiome data (ie, host genes, age, sex, diet, drugs) & No acknowledgement of potential confounders \\
\hline Longitudinal study design & Cross-sectional study design \\
\hline Large cohort & Small cohort \\
\hline Consistent storage and processing of samples & Ad hoc storage and processing of samples \\
\hline Accounting for potential contamination of low biomass samples & Processing low biomass samples in the same manner as high biomass samples \\
\hline Correction for multiple hypothesis testing & No correction for multiple hypothesis testing \\
\hline Publishing raw data and detailed methods (including code) for obtaining results & Not making raw data and detailed methods publicly available \\
\hline Interdisciplinary team & Lack of expertise \\
\hline Multiomic approach & Approach focused on 16S rRNA data \\
\hline Discovery and validation cohorts & Only discovery cohort \\
\hline Preserving samples & Discarding samples once study is complete \\
\hline
\end{tabular}

as environmental variables such as $\operatorname{diet}^{61}$ and drugs (even those without antibiotic or antimicrobial properties). ${ }^{62}$ In animal experiments, cage effects ${ }^{63}$ and facilities ${ }^{64}$ have a significant influence on the data and should be treated as separate variables of interest. Due to potential fluctuations in the microbiome, possibly related to disease flare/activity ${ }^{6566}$ and/or treatment, ${ }^{67}$ characterising the microbiome in a longitudinal fashion is of utmost importance (instead of assessing a single cross-sectional time point). Most importantly, many microbiome experiments are performed on small cohorts, leading to conclusions that are based on statistically invalid methodology. It is therefore critical to estimate adequate sample sizes a priori in order to sufficiently power these studies for the identification of biologically relevant patterns. This may be challenging given the lack of consensus on appropriate power analyses for microbiome studies. Nevertheless, several approaches have been proposed based on the detection of differences in $\alpha / \beta$ diversity and taxa relative abundance. ${ }^{68}$ Furthermore, the need to incorporate confounding variables typically necessitates larger sample sizes, which may be difficult to achieve, particularly in the case of the rarest conditions in rheumatology. An effective way to overcome this hurdle is through multicentre study designs and data sharing.

Once collected, samples should ideally be stored in a consistent fashion, processed at the same time with identical reagents and kits, and sequenced in single runs to avoid batch effect. The HMP Manual of Procedures provides detailed protocols for performing these steps. ${ }^{69}$ Positive and negative controls should always be used with every sequencing run. Low biomass samples, such as those derived from skin surface and lung fluid, present an extra challenge as they are prone to contamination from reagents and sensitive to sequencing conditions, thus requiring careful handling and the use of dedicated instruments. ${ }^{3}$ Postsequencing, certain software packages and computational methods may be employed to decrease background noise in low biomass data. In the analysis phase, comparisons are typically made across hundreds of identified taxa so multiple hypothesis testing must be accounted for by using statistical methods such as false discovery rate or Bonferroni corrections. ${ }^{70} 71$ Once results are obtained and published, the raw sequence data (including controls), a description of the detailed analyses, as well as any code written to generate results should be made publicly available through repositories such as the Sequence Read Archive, ${ }^{72}$ the European Nucleotide Archive ${ }^{73}$ and GitHub. ${ }^{74}$ This allows for cross-validation and incorporation of data in future studies.
Unused samples should also be stored to answer future questions that originate from current research efforts.

To produce more meaningful generalisable outcomes, it is essential to collaborate with an interdisciplinary team that can address questions of biological/clinical relevance, computational complexity and appropriate statistical models. Moreover, simply using DNA sequencing to look at the presence of taxa in certain conditions is now considered outdated and rather rudimentary. Cost permitting, the field should instead aim to design studies that explore the entire milieu of microbes (including bacteria, fungi and viruses, acknowledging recognised limitations such as the underdevelopment of comprehensive high-resolution reference databases for fungal and viral micro-organisms), microbemicrobe interactions, metabolites produced by microbes, and the effect that they have on the host immune and metabolic system. This requires a multipronged approach that applies shotgun and other multiomic sequencing platforms combined with more sophisticated computational analyses. Finally, generated results should be validated and reproduced in separate cohorts and backed preferentially by in vitro, ex vivo and in vivo data to look for recognisable patterns in order to enable a mechanistic understanding of the inflammatory process.

\section{Advances in microbiome research}

Despite these challenges, there have been pivotal advances in the oncology ${ }^{75}$ and IBD disciplines, ${ }^{76}$ where microbiome research has taken a more mechanistic approach beyond correlative descriptions. In fact, the gut microbiome is increasingly recognised for its influence on cancer and response to cancer therapy. ${ }^{77} 78$ For example, there are data supporting the notion that recurrent antibiotic use may be associated with the development of malignancies in various organs. ${ }^{79}$ However, the majority of studies that demonstrate a more causal role for the microbiome, aside from Helicobacter pylori-associated gastric cancer, ${ }^{80}$ are in colorectal cancer (CRC). First, microbial composition in tumor-affected tissue is distinct from that of adjacent healthy mucosa. ${ }^{81} 82$ Second, and even more striking is that transplanting stool from patients with CRC can cause the formation of polyps, increase levels of intestinal dysplasia and alter the local immune environment in animal models. ${ }^{83}$ Moreover, specific bacteria have been shown to directly stimulate inflammation and promote carcinogenesis, including enterotoxigenic Bacteroides fragilis ${ }^{84}$ and Fusobacterium nucleatum. ${ }^{85}$ The intestinal microbiota are also key drivers for patient response to therapy, as exemplified 
by immune checkpoint inhibitors used in the treatment of melanoma, ${ }^{86-88}$ where distinct differences exist between responders and non-responders that can be recapitulated in germ-free or antibiotic-treated animal models using faecal microbiota transplantation (FMT) from patients. ${ }^{89}$

In IBD, which includes Crohn's disease (CD) and ulcerative colitis (UC), gut microbial dysbiosis is apparent in both the reduction of important gut commensals and the expansion of pathogens. For example, a reduction of $F$. prausnitzii, an important producer of the SCFA butyrate (discussed earlier) is well documented in both $\mathrm{CD}^{90}$ and UC. ${ }^{91}$ Although specific bacterial pathobionts have not been consistently identified, there are indications of certain microbiota driving inflammation, ${ }^{92}$ as well as an increased prevalence of Enterobacteriaceae such as invasive Escherichia coli. ${ }^{93}$ Several studies highlight the role of fungi, including the expansion of Candida albicans, ${ }^{94} 95$ an immunogen for anti-Saccharomyces cerevisiae antibodies, which serve as serological markers in IBD. ${ }^{96}$ Even the virome has been implicated with the expansion of Caudovirales bacteriophages in patients with IBD. ${ }^{97}$ Furthermore, due to its dynamic nature, one study looked at the longitudinal composition of the gut microbiome, demonstrating that microbial communities in IBD exhibit more fluctuations compared with healthy individuals. ${ }^{98}$ Another study profiled and correlated the gut metagenomes and metatranscriptomes over a 1 year period, showing speciesspecific biases in transcriptional activity with a predominance of F. prausnitzii-associated pathways that were disproportionate to F. prausnitzii abudnance. ${ }^{99}$ A number of investigations have also looked at adaptive immune responses to microbial agents and have shown that patients with IBD produce large amounts of IgG antibodies ${ }^{100}$ and CD4+ T-lymphocytes with altered IL-17A production ${ }^{101}$ directed against symbiotic bacteria.

IBD investigators have led the way in FMT research, which gained widespread interest after its success in treating refractory Clostridioides difficile (previously named Clostridium difficile) infections. ${ }^{102} 103$ FMT involves the transfer of minimally manipulated prescreened stool from a donor to the gastrointestinal tract of a recipient via a nasogastric tube, colonoscopy, retention enema or capsule, with the aim of reversing a dysbiotic state and restoring beneficial microbiota. ${ }^{104}$ Four randomised controlled trials have been published looking at the efficacy of FMT in UC, ${ }^{105-108}$ showing an overall remission rate of $37 \%$, which was twofold higher than patients receiving placebo, ${ }^{109}$ and equivalent to outcomes with biological therapies (figure 1). ${ }^{110}$ To date, there have not been any randomised controlled trials of FMT use in $\mathrm{CD}$, although a recent meta-analysis reports an overall rate of clinical remission, ${ }^{111}$ a finding that may be less interpretable given the heterogeneity of patient disease activity and FMT protocols. ${ }^{104}$

\section{What the future holds: pharmacomicrobiomics and microbiome-modulating strategies in rheumatology}

The field of rheumatology has also made advances with the potential to significantly impact the care of our patients. One example is the growing field of pharmacomicrobiomics, which describes the effects that microbial variations have on the action and toxicity of drugs (and vice versa). ${ }^{12}$ For instance, we know that the activation of sulfasalazine, a disease-modifying antirheumatic drug used to treat inflammatory arthritis and UC, is dependent on the enzymatic cleavage by gut microbes. ${ }^{113}$ This appears to also hold true for methotrexate, which is known to be metabolised by the gut microbiome in mice ${ }^{114} 115$ and humans, ${ }^{116}$ and may have off-target, antibiotic effects. ${ }^{117}$ In axial
SpA, patients who respond to anti-TNF inhibitors exhibit a more resilient pretreatment gut microbiome, ${ }^{118}$ while IL-17A inhibitors are associated with expansion of intestinal C. albicans in a subgroup of paients with $\mathrm{SpA} / \mathrm{PsA},{ }^{67}$ as well as an increased risk for the development of candidiasis. ${ }^{119}{ }^{120}$ Further progress in pharmacomicrobiomics will lead towards personalised therapeutic approaches that are based on patient microbiome features, allowing for improved selection of medications with the highest efficacy and lowest risk for toxicity. ${ }^{121}$

Another promising area of active research is the study of targeted modulation of the microbiome to improve disease outcomes, with the caveat that for most conditions, it is not yet clear whether microbial changes contribute to disease pathogenesis or stem from the disease process itself. One indirect way to modulate the microbiome is through diet, which can globally shape the microbial community composition. Few conclusive studies exist on the topic but some trials looking at the Mediterranean diet have demonstrated beneficial effects in RA. ${ }^{122} 123$ In PsA, which is strongly associated with obesity and metabolic syndrome, weight loss has also led to significant improvements in disease outcomes. ${ }^{124} 125$ Another strategy relies on the use of prebiotics, compounds that promote the growth of advantageous microbes (ie, SCFAs and MCFAs), and probiotics, compounds that contain presumed beneficial living organisms. Prebiotics have demonstrated value in an animal model of SpA, ${ }^{126}$ while probiotics containing Lactobacillus have been associated with improvements in RA disease activity scores. ${ }^{127-129}$ As discussed, a more invasive approach that has yielded encouraging results is FMT, which directly modifies microbial communities and their metabolites, and may directly or indirectly stimulate the host immune response. No conclusive evidence exists in rheumatic disease as of yet, but this is currently being studied in PsA by the Danish-sponsored Efficacy and Safety of Fecal Microbiota Transplantation in Peripheral Psoriatic Arthritis clinical trial (figure 1). ${ }^{130}$ However, it is important to recognise that FMT has several important limitations. For instance, we do not know the best route of delivery (oral vs rectal) or the frequency of FMT needed to achieve durable responses. Likewise, we have not identified specific beneficial taxa that can reliably attenuate various autoimmune conditions, and it appears that previous successes in IBD may have been driven by 'super-donors' (ie, donors whose stool samples achieved successful outcomes). ${ }^{131}$ Extensive donor testing is also required to guarantee safety as recent reports have indicated transmission of drug-resistant organisms from donors to recipients. ${ }^{132}{ }^{133}$ Aside from FMT, other potential strategies, which are being extensively tested by the pharmaceutical industry but are yet to be Food and Drug Administration approved, are microbial consortia or microbially derived bioactive compounds for the treatment of infectious and autoimmune diseases. ${ }^{134}$

\section{CONCLUSIONS}

Microbiome research in rheumatology is expanding significantly, offering unique opportunities to better understand aspects of autoimmune disease pathogenesis, the potential for patient stratification and its application towards personalised therapeutic strategies. However, in order to achieve its full potential, there is a need to further adapt state-of-the-art microbiome-related methods and technologies in our discipline. It is imperative to continue the quest for biologically relevant inquiries, addressing causality and designing studies that are important to patient care. The expansion of collaborations and data sharing is also critical to increase the sample size of our cohorts and apply the 
appropriate expertise. It is equally important to broaden the scope of these studies to look at the global network of organisms, their interactions and what they produce, so we can move beyond simplistic taxonomic classification work. Finally, the validation of microbiome-derived data should ensure meaningful results that could potentially serve in diagnostics and therapeutics, aiding in the development of precision medicine approaches to optimise health outcomes in rheumatic and autoimmune diseases.

Contributors All authors have contributed to the conception of the work, drafting and revising the work, and final approval of the version to be published.

Funding JUS is funded by NIH/NIAMS R01AR074500, the National Psoriasis Foundation, the Colton Center for Autoimmunity, the Riley Family Foundation and the Snyder Family Foundation.

Competing interests JUS has been granted USPTO patent no. 10011883 ('Causative agents and diagnostic methods relating to rheumatoid arthritis'). JUS has consulted for UCB, Janssen, Novartis and Pfizer.

Patient and public involvement Patients and/or the public were not involved in the design, or conduct, or reporting, or dissemination plans of this research.

Patient consent for publication Not required.

Provenance and peer review Commissioned; externally peer reviewed.

\section{ORCID iDs}

Julia Manasson http://orcid.org/0000-0003-3448-5520

Jose U Scher http://orcid.org/0000-0002-1072-6994

\section{REFERENCES}

1 Lederberg J, McCray AT. Ome Sweet 'Omics - A Genealogical Treasury of Words. Scientist 2001:15:8

2 Clemente JC, Manasson J, Scher JU. The role of the gut microbiome in systemic inflammatory disease. BMJ 2018:360::5145.

3 Kim D, Hofstaedter CE, Zhao C, et al. Optimizing methods and dodging pitfalls in microbiome research. Microbiome 2017:5:52

4 Eckburg PB, Bik EM, Bernstein CN, et al. Diversity of the human intestinal microbial flora. Science 2005;308:1635-8.

5 Aguiar-Pulido V, Huang W, Suarez-Ulloa V, et al. Metatranscriptomics, and metabolomics approaches for microbiome analysis. Evol Bioinform Online 2016;12:5-16.

6 Siggins A, Gunnigle E, Abram F. Exploring mixed microbial community functioning: recent advances in metaproteomics. FEMS Microbiol Ecol 2012;80:265-80

7 Turnbaugh PJ, Ley RE, Hamady M, et al. The human microbiome project. Nature 2007:449:804-10.

8 Qin J, Li R, Raes J, et al. A human gut microbial gene Catalogue established by metagenomic sequencing. Nature 2010;464:59-65.

9 Rosenbaum JT, Asquith MJ. The microbiome: a revolution in treatment for rheumatic diseases? Curr Rheumatol Rep 2016;18:62.

10 Mancini N, Peri F, Rescigno M, et al. Microbiome studies in the medical sciences and the need for closer multidisciplinary interplay. Sci Signal 2020;13:eaba9911.

11 Taurog JD, Richardson JA, Croft JT, et al. The germfree state prevents development of gut and joint inflammatory disease in HLA-B27 transgenic rats. J Exp Med 1994; 180:2359-64.

12 Abdollahi-Roodsaz $\mathrm{S}$, Joosten LAB, Koenders MI, et al. Stimulation of TLR2 and TLR4 differentially skews the balance of $\mathrm{T}$ cells in a mouse model of arthritis. I Clin Invest 2008:118:205-16.

13 HJ W, Ivanov, II DJ, et al. Gut-residing segmented filamentous bacteria drive autoimmune arthritis via T helper 17 cells. Immunity 2010;32:815-27.

14 Yoshitomi H, Sakaguchi N, Kobayashi K, et al. A role for fungal $\beta$-glucans and their receptor Dectin-1 in the induction of autoimmune arthritis in genetically susceptible mice. J Exp Med 2005:201:949-60.

15 Honda K, Littman DR. The microbiome in infectious disease and inflammation. Annu Rev Immunol 2012:30:759-95.

16 Osler W. The principles and practice of medicine, designed for the use of practitioners and students of medicine. 6th edn. New York, London: D. Appleton and company, 1905.

17 Warden CC. The toxemic factor in rheumatoid arthritis. Cal State J Med 1909;7:299-301

18 Dunbar LL. Oral manifestations in arthrititic and gouty conditions. read in the section on dental and oral surgery atF-fAMotAMA, held at San Francisco, June 5-8, 1894. JAMA 1895;XXIV:75-7.

19 Scher JU, Ubeda C, Equinda M, et al. Periodontal disease and the oral microbiota in new-onset rheumatoid arthritis. Arthritis Rheum 2012;64:3083-94.
20 Hitchon CA, Chandad F, Ferucci ED, et al. Antibodies to Porphyromonas gingivalis Are Associated with Anticitrullinated Protein Antibodies in Patients with Rheumatoid Arthritis and Their Relatives. I Rheumatol 2010;37:1105-12.

21 Mikuls TR, Payne JB, Reinhardt RA, et al. Antibody responses to Porphyromonas gingivalis (P. gingivalis) in subjects with rheumatoid arthritis and periodontitis. Int Immunopharmacol 2009:9:38-42.

22 Halvorsen EH, Pollmann S, Gilboe I-M, et al. Serum IgG antibodies to peptidylarginine deiminase 4 in rheumatoid arthritis and associations with disease severity. Ann Rheum Dis 2008:67:414-7.

23 Harris ML, Darrah E, Lam GK, et al. Association of autoimmunity to peptidyl arginine deiminase type 4 with genotype and disease severity in rheumatoid arthritis. Arthritis Rheum 2008;58:1958-67.

24 Darrah E, Giles JT, Ols ML, et al. Erosive rheumatoid arthritis is associated with antibodies that activate PAD4 by increasing calcium sensitivity. Sci Trans/ Med 2013;5:186ra65.

25 Giles JT, Darrah E, Danoff S, et al. Association of cross-reactive antibodies targeting peptidyl-arginine deiminase 3 and 4 with rheumatoid arthritis-associated interstitial lung disease. PLoS One 2014;9:e98794.

26 Scher JU, Sczesnak A, Longman RS, et al. Expansion of intestinal Prevotella copri correlates with enhanced susceptibility to arthritis. elife 2013;2:e01202.

27 Maeda Y, Kurakawa T, Umemoto E, et al. Dysbiosis contributes to arthritis development via activation of autoreactive T cells in the intestine. Arthritis Rheumatol 2016;68:2646-61.

28 Pianta A, Arvikar S, Strle K, et al. Evidence of the immune relevance of Prevotella copri, a gut microbe, in patients with rheumatoid arthritis. Arthritis Rheumatol 2017:69:964-75.

29 Lin P, Bach M, Asquith M, et al. Hla-B27 and human $\beta 2$-microglobulin affect the gut microbiota of transgenic rats. PLoS One 2014:9:e105684.

30 Asquith MJ, Stauffer P, Davin S, et al. Perturbed mucosal immunity and dysbiosis accompany clinical disease in a rat model of spondyloarthritis. Arthritis Rheumatol 2016;68:2151-62.

31 Gill T, Asquith M, Brooks SR, et al. Effects of HLA-B27 on gut microbiota in experimental spondyloarthritis implicate an ecological model of dysbiosis. Arthritis Rheumatol 2018;70:555-65

32 Fiessinger N, Leroy E. Contribution l'étude d'une épidémie de dysenterie dans La Somme, bull. et mém. Soc méd hôp Paris 1916;40:2030.

33 García-Kutzbach A, Chacón-Súchite J, García-Ferrer H, et al. Reactive arthritis: update 2018. Clin Rheumatol 2018;37:869-74.

34 Bargen JA JR, Kerr JG. Complications and sequel of chronic ulcerative colitis. Annals of Internal Medicine 1929;3:335-52.

35 Peluso R, Di Minno MND, lervolino S, et al. Enteropathic spondyloarthritis: from diagnosis to treatment. Clin Dev Immunol 2013;2013:1-12.

36 Costello M-E, Ciccia F, Willner D, et al. Brief report: intestinal dysbiosis in ankylosing spondylitis. Arthritis Rheumatol 2015;67:686-91.

37 Cao Y, Shen J, Ran ZH. Association between Faecalibacterium prausnitzii reduction and inflammatory bowel disease: a meta-analysis and systematic review of the literature. Gastroenterol Res Pract 2014;2014:1-7.

38 Hold GL, Schwiertz A, Aminov Rl, et al. Oligonucleotide probes that detec quantitatively significant groups of butyrate-producing bacteria in human feces. App Environ Microbiol 2003:69:4320-4.

39 Furusawa Y, Obata Y, Fukuda S, et al. Commensal microbe-derived butyrate induces the differentiation of colonic regulatory T cells. Nature 2013;504:446-50

40 Scher JU, Ubeda C, Artacho A, et al. Decreased bacterial diversity characterizes the altered gut microbiota in patients with psoriatic arthritis, resembling dysbiosis in inflammatory bowel disease. Arthritis Rheumatol 2015:67:128-39.

41 Hevia A, Milani C, López P, et al. Intestinal dysbiosis associated with systemic lupus erythematosus. MBio 2014:5:e01548-14.

42 Bellocchi C, Fernández-Ochoa Álvaro, Montanelli G, et al. Identification of a shared microbiomic and metabolomic profile in systemic autoimmune diseases. $J$ Clin Med 2019:8:1291.

43 López P, de Paz B, Rodríguez-Carrio J, et al. Th17 responses and natural IgM antibodies are related to gut microbiota composition in systemic lupus erythematosus patients. Sci Rep 2016;6:24072.

44 Li Y, Wang H-F, Li X, et al. Disordered intestinal microbes are associated with the activity of systemic lupus erythematosus. Clin Sci 2019:133:821-38.

$45 \mathrm{He}$ Z, Shao T, Li H, et al. Alterations of the gut microbiome in Chinese patients with systemic lupus erythematosus. Gut Pathog 2016;8:64.

46 Luo XM, Edwards MR, Mu Q, et al. Gut microbiota in human systemic lupus erythematosus and a mouse model of lupus. Appl Environ Microbiol 2018;84

47 van der Meulen TA, Harmsen HJM, Vila AV, et al. Shared gut, but distinct oral microbiota composition in primary Sjögren's syndrome and systemic lupus erythematosus. J Autoimmun 2019:97:77-87.

48 Azzouz D, Omarbekova A, Heguy A, et al. Lupus nephritis is linked to diseaseactivity associated expansions and immunity to a gut commensal. Ann Rheum Dis 2019:78:947-56.

49 Manfredo Vieira S, Hiltensperger M, Kumar V, et al. Translocation of a gut pathobiont drives autoimmunity in mice and humans. Science 2018;359:1156-61 
50 Greiling TM, Dehner C, Chen X, et al. Commensal orthologs of the human autoantigen Ro60 as triggers of autoimmunity in lupus. Sci Trans/ Med 2018; 10:eaan2306.

51 Andréasson K, Alrawi Z, Persson A, et al. Intestinal dysbiosis is common in systemic sclerosis and associated with gastrointestinal and extraintestinal features of disease. Arthritis Res Ther 2016;18:278.

52 Volkmann ER, Chang Y-L, Barroso N, et al. Association of systemic sclerosis with a unique colonic microbial consortium. Arthritis Rheumatol 2016:68:1483-92.

53 Volkmann ER, Hoffmann-Vold A-M, Chang Y-L, et al. Systemic sclerosis is associated with specific alterations in gastrointestinal microbiota in two independent cohorts. BMJ Open Gastroenterol 2017;4:e000134.

54 Bellocchi C, Fernández-Ochoa Álvaro, Montanelli G, et al. Microbial and metabolic multi-omic correlations in systemic sclerosis patients. Ann N Y Acad Sci 2018;1421:97-109

55 Patrone V, Puglisi E, Cardinali M, et al. Gut microbiota profile in systemic sclerosis patients with and without clinical evidence of gastrointestinal involvement. Sci Rep 2017;7:14874.

56 Asquith M, Sternes PR, Costello M-E, et al. HLA Alleles Associated With Risk of Ankylosing Spondylitis and Rheumatoid Arthritis Influence the Gut Microbiome. Arthritis Rheumatol 2019;71:1642-50.

57 Manasson J, Shen N, Garcia Ferrer HR, et al. Gut microbiota perturbations in reactive arthritis and postinfectious spondyloarthritis. Arthritis Rheumatol 2018;70:242-54

58 Yatsunenko T, Rey FE, Manary MJ, et al. Human gut microbiome viewed across age and geography. Nature 2012;486:222-7.

59 Claesson MJ, Cusack S, O'Sullivan 0, et al. Composition, variability, and temporal stability of the intestinal microbiota of the elderly. Proc Natl Acad Sci U SA 2011;108:4586-91.

60 Markle JGM, Frank DN, Mortin-Toth S, et al. Sex differences in the gut microbiome drive hormone-dependent regulation of autoimmunity. Science 2013:339:1084-8.

61 Rothschild D, Weissbrod O, Barkan E, et al. Environment dominates over host genetics in shaping human gut microbiota. Nature 2018;555:210-5.

62 Maier L, Pruteanu M, Kuhn M, et al. Extensive impact of non-antibiotic drugs on human gut bacteria. Nature 2018;555:623-8.

63 Hildebrand F, Nguyen TLA, Brinkman B, et al. Inflammation-Associated enterotypes, host genotype, cage and inter-individual effects drive gut microbiota variation in common laboratory mice. Genome Biol 2013;14:R4.

64 Parker KD, Albeke SE, Gigley JP, et al. Microbiome composition in both wild-type and disease model mice is heavily influenced by mouse facility. Front Microbiol 2018:9:1598

65 Braun T, Di Segni A, BenShoshan M, et al. Individualized dynamics in the gut microbiota precede Crohn's disease flares. Am J Gastroenterol 2019;114:1142-51.

66 Lloyd-Price J, Arze C, Ananthakrishnan AN, et al. Multi-Omics of the gut microbial ecosystem in inflammatory bowel diseases. Nature 2019;569:655-62.

67 Manasson J, Wallach DS, Guggino G, et al. II-17 inhibition in spondyloarthritis associates with subclinical gut microbiome perturbations and a distinctive IL-25Driven intestinal inflammation. Arthritis Rheumatol 2019.

$68 \mathrm{CI}$ L, Samuels DC, Zhao YY, et al. Power and sample size calculations for highthroughput sequencing-based experiments. Brief Bioinform 2018;19:1247-55.

69 Mclnnes P, Cutting M. Core Microbiome Sampling Protocol A - HMP Protocol \#07001, version 12.0. Manual of Procedures for the Human Microbiome Project 2010.

70 Benjamini Y, Hochberg Y. Controlling the false discovery rate: a practical and powerful approach to multiple testing. Journal of the Royal Statistical Society: Series B 1995:57:289-300.

71 Bland JM, Altman DG. Multiple significance tests: the Bonferroni method. BMJ 1995;310:170.

72 Leinonen $R$, Sugawara $H$, Shumway M. International nucleotide sequence database C. the sequence read archive. Nucleic Acids Res 2011;39:D19-21.

73 Leinonen R, Akhtar R, Birney E, et al. The European nucleotide Archive. Nucleic Acids Res 2011;39:D28-31.

74 Blischak JD, Davenport ER, Wilson G. A quick introduction to version control with GIT and GitHub. PLoS Comput Biol 2016;12:e1004668.

75 Helmink BA, Khan MAW, Hermann A, et al. The microbiome, cancer, and cancer therapy. Nat Med 2019;25:377-88.

76 Caruso R, Lo BC, Núñez G. Host-microbiota interactions in inflammatory bowel disease. Nat Rev Immunol 2020;12.

77 Alexander JL, Wilson ID, Teare J, et al. Gut microbiota modulation of chemotherapy efficacy and toxicity. Nat Rev Gastroenterol Hepatol 2017;14:356-65.

78 Garrett WS. Cancer and the microbiota. Science 2015;348:80-6.

79 Boursi B, Mamtani R, Haynes K, et al. Recurrent antibiotic exposure may promote cancer formation - another step in understanding the role of the human microbiota? Eur J Cancer 2015;51:2655-64.

80 Wang F, Meng W, Wang B, et al. Helicobacter pylori-induced gastric inflammation and gastric cancer. Cancer Lett 2014;345:196-202.

81 Nakatsu G, Li X, Zhou H, et al. Gut mucosal microbiome across stages of colorectal carcinogenesis. Nat Commun 2015;6:8727.

82 Lu Y, Chen J, Zheng J, et al. Mucosal adherent bacterial dysbiosis in patients with colorectal adenomas. Sci Rep 2016;6:26337.
83 Wong SH, Zhao L, Zhang X, et al. Gavage of Fecal Samples From Patients With Colorectal Cancer Promotes Intestinal Carcinogenesis in Germ-Free and Conventional Mice. Gastroenterology 2017;153:1621-33.

84 Wu S, Rhee K-J, Albesiano E, et al. A human colonic commensal promotes colon tumorigenesis via activation of T helper type $17 \mathrm{~T}$ cell responses. Nat Med 2009;15:1016-22.

85 Kostic AD, Chun E, Robertson L, et al. Fusobacterium nucleatum potentiates intestinal tumorigenesis and modulates the tumor-immune microenvironment. Cell Host Microbe 2013;14:207-15.

86 Frankel AE, Coughlin LA, Kim J, et al. Metagenomic shotgun sequencing and unbiased metabolomic profiling identify specific human gut microbiota and metabolites associated with immune checkpoint therapy efficacy in melanoma patients. Neoplasia 2017:19:848-55.

87 Matson V, Fessler J, Bao R, et al. The commensal microbiome is associated with antiPD-1 efficacy in metastatic melanoma patients. Science 2018:359:104-8.

88 Gopalakrishnan V, Spencer CN, Nezi L, et al. Gut microbiome modulates response to anti-PD-1 immunotherapy in melanoma patients. Science 2018;359:97-103.

89 Routy B, Le Chatelier E, Derosa L, et al. Gut microbiome influences efficacy of PD-1based immunotherapy against epithelial tumors. Science 2018;359:91-7.

90 Takahashi K, Nishida A, Fujimoto T, et al. Reduced abundance of butyrate-producing bacteria species in the fecal microbial community in Crohn's disease. Digestion 2016;93:59-65.

91 Machiels K, Joossens M, Sabino J, et al. A decrease of the butyrate-producing species Roseburia hominis and Faecalibacterium prausnitzii defines dysbiosis in patients with ulcerative colitis. Gut 2014;63:1275-83.

92 Xavier RJ, Podolsky DK. Unravelling the pathogenesis of inflammatory bowel disease. Nature 2007;448:427-34.

93 Pickard JM, Zeng MY, Caruso R, et al. Gut microbiota: role in pathogen colonization, immune responses, and inflammatory disease. Immunol Rev 2017;279:70-89.

94 Li Q, Wang C, Tang C, et al. Dysbiosis of gut fungal microbiota is associated with mucosal inflammation in Crohn's disease. J Clin Gastroenterol 2014;48:513-23.

95 Sokol H, Leducq V, Aschard H, et al. Fungal microbiota dysbiosis in IBD. Gut 2017:66:1039-48.

96 Standaert-Vitse A, Jouault T, Vandewalle P, et al. Candida albicans Is an Immunogen for Anti-Saccharomyces cerevisiae Antibody Markers of Crohn's Disease. Gastroenterology 2006;130:1764-75

97 Norman JM, Handley SA, Baldridge MT, et al. Disease-Specific alterations in the enteric virome in inflammatory bowel disease. Cell 2015;160:447-60.

98 Halfvarson J, Brislawn CJ, Lamendella R, et al. Dynamics of the human gut microbiome in inflammatory bowel disease. Nat Microbiol 2017;2:17004.

99 Schirmer M, Franzosa EA, Lloyd-Price J, et al. Dynamics of metatranscription in the inflammatory bowel disease gut microbiome. Nat Microbiol 2018;3:337-46.

100 Macpherson A, Khoo UY, Forgacs I, et al. Mucosal antibodies in inflammatory bowel disease are directed against intestinal bacteria. Gut 1996;38:365-75.

101 Hegazy AN, West NR, Stubbington MJT, et al. Circulating and tissue-resident CD4 ${ }^{+}$T Cells with reactivity to intestinal microbiota are abundant in healthy individuals and function is altered during inflammation. Gastroenterology 2017;153:e16:1320-37.

102 van Nood E, Vrieze A, Nieuwdorp M, et al. Duodenal Infusion of Donor Feces for Recurrent Clostridium difficile. N Engl J Med 2013;368:407-15.

103 Cammarota G, Masucci L, laniro G, et al. Randomised clinical trial: faecal microbiota transplantation by colonoscopy vs. vancomycin for the treatment of recurrent Clostridium difficile infection. Aliment Pharmacol Ther 2015;41:835-43.

104 Allegretti JR, Mullish BH, Kelly C, et al. The evolution of the use of faecal microbiota transplantation and emerging therapeutic indications. Lancet 2019;394:420-31.

105 Rossen NG, Fuentes S, van der Spek MJ, et al. Findings from a randomized controlled trial of fecal transplantation for patients with ulcerative colitis. Gastroenterology 2015;149:110-8

106 Moayyedi P, Surette MG, Kim PT, et al. Fecal microbiota transplantation induces remission in patients with active ulcerative colitis in a randomized controlled trial. Gastroenterology 2015;149:102-9.

107 Paramsothy S, Kamm MA, Kaakoush NO, et al. Multidonor intensive faecal microbiota transplantation for active ulcerative colitis: a randomised placebocontrolled trial. Lancet 2017;389:1218-28.

108 Costello SP, Hughes PA, Waters O, et al. Effect of fecal microbiota transplantation on 8-Week remission in patients with ulcerative colitis: a randomized clinical trial. JAMA 2019:321:156-64.

109 Imdad A, Nicholson MR, Tanner-Smith EE, et al. Fecal transplantation for treatment of inflammatory bowel disease. Cochrane Database Syst Rev 2018:11:CD012774.

110 Kelly CR, Ananthakrishnan AN. Manipulating the microbiome with fecal transplantation to treat ulcerative colitis. JAMA 2019:321:151-2.

111 Paramsothy S, Paramsothy R, Rubin DT, et al. Faecal microbiota transplantation for inflammatory bowel disease: a systematic review and meta-analysis. J Crohns Colitis 2017;11:1180-99.

112 Rizkallah M, Saad R, Aziz R. The human microbiome project, personalized medicine and the birth of pharmacomicrobiomics. Curr Pharmacogenomics Person Med 2010:8:182-93

113 Peppercorn MA, Goldman P. The role of intestinal bacteria in the metabolism of salicylazosulfapyridine. J Pharmacol Exp Ther 1972;181:555-62. 
114 Zaharko DS, Bruckner H, Oliverio VT. Antibiotics alter methotrexate metabolism and excretion. Science 1969;166:887-8.

115 Valerino DM, Johns DG, Zaharko DS, et al. Studies of the metabolism of methotrexate by intestinal flora. I. Identification and study of biological properties of the metabolite 4-amino-4-deoxy-N 10 -methylpteroic acid. Biochem Pharmacol 1972;21:821-31

116 Isaac SAA, Nayak R, Abramson S, et al. The pre-treatment gut microbiome predicts early response to methotrexate in Rheumatoid Arthritis [abstract]. Arthritis Rheumatol 2019;71.

117 Nayak RR, Alexander M, Stapleton-Grey K, et al. Perturbation of the human gut microbiome by a non-antibiotic drug contributes to the resolution of autoimmune disease. bioRxiv 2019:600155.

118 Bazin T, Hooks KB, Barnetche T, et al. Microbiota composition may predict antiTNF alpha response in spondyloarthritis patients: an exploratory study. Sci Rep 2018;8:5446

119 Mclnnes IB, Mease PJ, Kirkham B, et al. Secukinumab, a human antiinterleukin-17A monoclonal antibody, in patients with psoriatic arthritis (future 2): a randomised, double-blind, placebo-controlled, phase 3 trial. Lancet 2015:386:1137-46.

120 Mease P, Roussou E, Burmester GR, et al. Safety of ixekizumab in patients with psoriatic arthritis: results from a pooled analysis of three clinical trials. Arthritis Care Res 2018.

121 Scher JU, Nayak RR, Ubeda C, et al. Pharmacomicrobiomics in inflammatory arthritis: gut microbiome as modulator of therapeutic response. Nat Rev Rheumato/ 2020. doi:10.1038/s41584-020-0395-3. [Epub ahead of print: $10 \mathrm{Ma}$ 2020].

122 McKellar G, Morrison E, McEntegart A, et al. A pilot study of a Mediterranean-type diet intervention in female patients with rheumatoid arthritis living in areas of social deprivation in Glasgow. Ann Rheum Dis 2007;66:1239-43.

123 Sköldstam L, Hagfors L, Johansson G. An experimental study of a Mediterranean diet intervention for patients with rheumatoid arthritis. Ann Rheum Dis 2003;62:208-14.
124 Klingberg E, Bilberg A, Björkman S, et al. Weight loss improves disease activity in patients with psoriatic arthritis and obesity: an interventional study. Arthritis Res Ther 2019;21:17.

125 Di Minno MND, Peluso R, lervolino S, et al. Weight loss and achievement of minimal disease activity in patients with psoriatic arthritis starting treatment with tumour necrosis factor $\alpha$ blockers. Ann Rheum Dis 2014:73:1157-62

126 Asquith M, Davin S, Stauffer P, et al. Intestinal metabolites are profoundly altered in the context of HLA-B27 expression and functionally modulate disease in a rat model of spondyloarthritis. Arthritis Rheumatol 2017;69:1984-95.

127 Alipour B, Homayouni-Rad A, Vaghef-Mehrabany E, et al. Effects of Lactobacillus casei supplementation on disease activity and inflammatory cytokines in rheumatoid arthritis patients: a randomized double-blind clinical trial. Int J Rheum Dis 2014:17:519-27.

128 Zamani B, Golkar HR, Farshbaf S, et al. Clinical and metabolic response to probiotic supplementation in patients with rheumatoid arthritis: a randomized, double-blind, placebo-controlled trial. Int J Rheum Dis 2016;19:869-79.

129 Hatakka K, Martio J, Korpela M, et al. Effects of probiotic therapy on the activity and activation of mild rheumatoid arthritis-a pilot study. Scand J Rheumatol 2003:32:211-5.

130 Kragsnaes MS, Kjeldsen J, Horn HC, et al. Efficacy and safety of faecal microbiota transplantation in patients with psoriatic arthritis: protocol for a 6-month, doubleblind, randomised, placebo-controlled trial. BMJ Open 2018;8:e019231.

131 Wilson BC, Vatanen T, Cutfield WS, et al. The Super-Donor phenomenon in fecal microbiota transplantation. Front Cell Infect Microbiol 2019:9:2.

132 DeFilipp Z, Bloom PP, Torres Soto M, et al. Drug-Resistant E. coli Bacteremia Transmitted by Fecal Microbiota Transplant. N Engl J Med 2019:381:2043-50.

133 Blaser MJ. Fecal Microbiota Transplantation for Dysbiosis - Predictable Risks. N Engl J Med 2019:381:2064-6.

134 Abdollahi-Roodsaz S, Abramson SB, Scher JU. The metabolic role of the gut microbiota in health and rheumatic disease: mechanisms and interventions. Nat Rev Rheumatol 2016:12:446-55. 